\title{
Review of: "Physician-directed genetic screening to evaluate personal risk for medically actionable disorders: a large multi-center cohort study"
}

\author{
Brian Hon Yin Chung ${ }^{1}$ \\ 1 University of Hong Kong
}

Potential competing interests: The author(s) declared that no potential competing interests exist.

This paper "Physician-directed genetic screening to evaluate personal risk for medically actionable disorders: a large multi-center cohort study" is a very first publication using NGS panel to screen the health risk proactively in a large cohort of 10,478 adults. $15.5 \%$ carries a risk of actionable monogenic disorder, while 3.1 to $5.2 \%$ had clinically reportable variants based on ACMG secondary findings v2.0, and $2.0 \%$ had reportable variants in CDC Tier 1 conditions. This paper seems more like a white paper by Invitae to promote their product, given the 1st author and last author are also from Invitae, here are our consolidated thoughts:

1) They over-emphasized the benefit of physician-directed genetic screening without properly addressing all the potential risks. While they did address obvious limitations such as gene penetrance and the lack of genetic expertise, they neglected the equally important issues such as possible genetic discrimination in terms of insurance/employment, psychological harm/emotional burden created that could potentially expand to other family members in case of any incidental findings. In addition, since they have used a more specific approach (page 7 discussion, third paragraph) in reporting/classifying variants, there are possibilities of false-negatives. As a result, we also need to educate the public that having a negative report does not mean there is no substantial genetic risk, especially since the screening is only a targeted panel.

2) Because this cohort is physician directed, there will be an unavoidable selection bias, where participants could be convenient samples who seek medical services, as can be seen from the cohort demographics: majority subjects were female between age 40-59 who might more often seek medical than other age group/sex.

3) In regards to variant reclassification, it will be even more helpful to illustrate with some case examples as to which variants have been upgraded/downgraded and their subsequent clinical implication to further demonstrate the impact. 
4) They mentioned the use of a "phenotype risk score" in which phenotype-genotype associations were used to identify patients with 5 mendelian disorders that had previously been undiagnosed or diagnosed incorrectly. It will be interesting to learn more and see if it will be applicable to us.

5) This study covered a period of 4 years. It will be interesting to have a longitudinal follow up to see how these genetic findings impact these patients in terms of long term clinical outcome/benefit/any behavioural changes.

In summary, this is a good paper for physicians to have a broad overview of the benefit of genetic screening; however, one should be reminded that there are also other potential risks involved that are not being mentioned in the paper 